\title{
Complete Analysis of Samples using Complementary Techniques
}

\author{
V.S. Smentkowski, D. Wark, L. Le Tarte, H. Piao, J.C. Chera, S.G. Ostrowski and A. \\ Suzuki \\ GE Global Research, Niskayuna, NY 12309
}

A combination of complimentary analysis techniques has been used to characterize a diffusion couple sample. We will demonstrate that the results, when taken together, provide for a better understanding of the sample than the data from any one technique alone. The poster will summarize how diffusion couples are fabricated and highlight the benefits of each characterization technique. Electron Probe Micro Analysis (EPMA) provides for quantitative chemical analysis (spectrometry and imaging) of most elements $(Z>4)$ at a concentration greater than about $0.1 \%$ within a volume of $1-2$ microns. FIG 1 shows EPMA line scans that were generated for $\mathrm{O}, \mathrm{Ni}, \mathrm{Cr}$, and $\mathrm{Mn}$; $\mathrm{O}$ is below the detection limit of EPMA in this sample. EPMA also allows for imaging of both backscattered and secondary electrons. Auger Electron Spectroscopy (AES) has the smallest analytical spot, provides for quantitative analysis of all elements (except $\mathrm{H}$ and $\mathrm{Li}$ ) present at concentrations of greater than about 0.5 atomic percent, and can perform high lateral resolution imaging. FIG 2 shows AES spectra representative of O (top), Mn (middle) and $\mathrm{Cr}$ (bottom), note that the oxygen AES peak occurs at the same energy as the Mn peak and prevents the identification of trace amounts of oxygen. X-Ray Photoelectron Spectroscopy (XPS) provides for quantitative analysis of all elements (except $\mathrm{H}$ and $\mathrm{Li}$ ) present at concentrations greater than about 0.5 atomic percent, is able to analyze insulating samples, and can provide information regarding the chemical state of the material. FIG 3 shows an XPS depth profile measurement. Oxygen is detected on the outer surface of the sample, however rapidly decreases to zero indicating that the amount of oxygen in the sample is less than about $100 \mathrm{ppm}$. Time of Flight Secondary Ion Mass Spectrometry (ToF-SIMS) is able to detect all elements and high mass molecular fragment ions with high sensitivity, is able to analyze non-conductive samples, and the analyst does not need to select which species to analyze for prior to the measurement since a full mass spectrum is saved at every volume element. FIG 4 shows ToF-SIMS images measured on the same sample that was analyzed by EPMA, AES, and XPS (left) as well as an image that was measured on a control sample (right). These ToF-SIMS images were collected after performing in-situ DC sputter cleaning to remove the surface contaminant layer. The control sample revealed significantly less oxygen than the treated sample. The concentration of oxygen in the treated sample is uniformly distributed in the lateral dimension. Each of the surface analysis instruments (AES, XPS, and ToF-SIMS) can operate in spectrometry modes (compositional analysis), imaging modes, as well as depth profiling modes (where one monitors the sample composition as a function of depth). In this poster, we will demonstrate that certain species reveal high ToF-SIMS spectral intensities even when present at low (ppm) concentrations. 


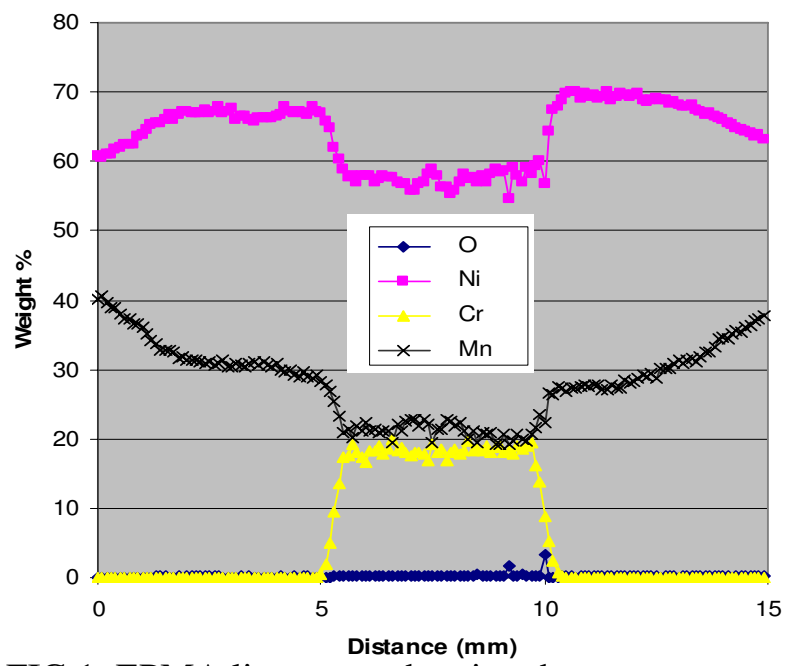

FIG 1. EPMA line scans showing the concentration of $\mathrm{O}, \mathrm{Ni}, \mathrm{Cr}$, and $\mathrm{Mn}$ across the region of interest.

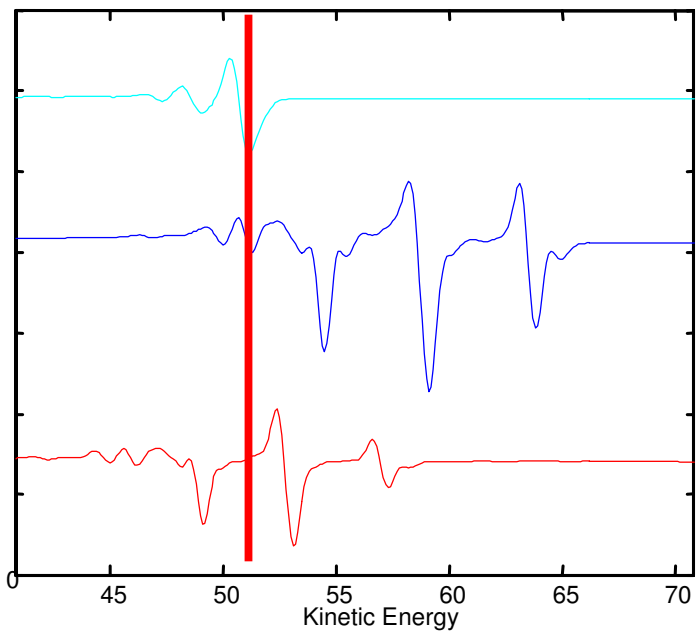

FIG 2. AES reference spectra. Top = oxygen middle $=\mathrm{Mn}$, and bottom $=\mathrm{Cr}$. The red line Reveals overlap between $\mathrm{O}$ and $\mathrm{Mn}$ peaks.

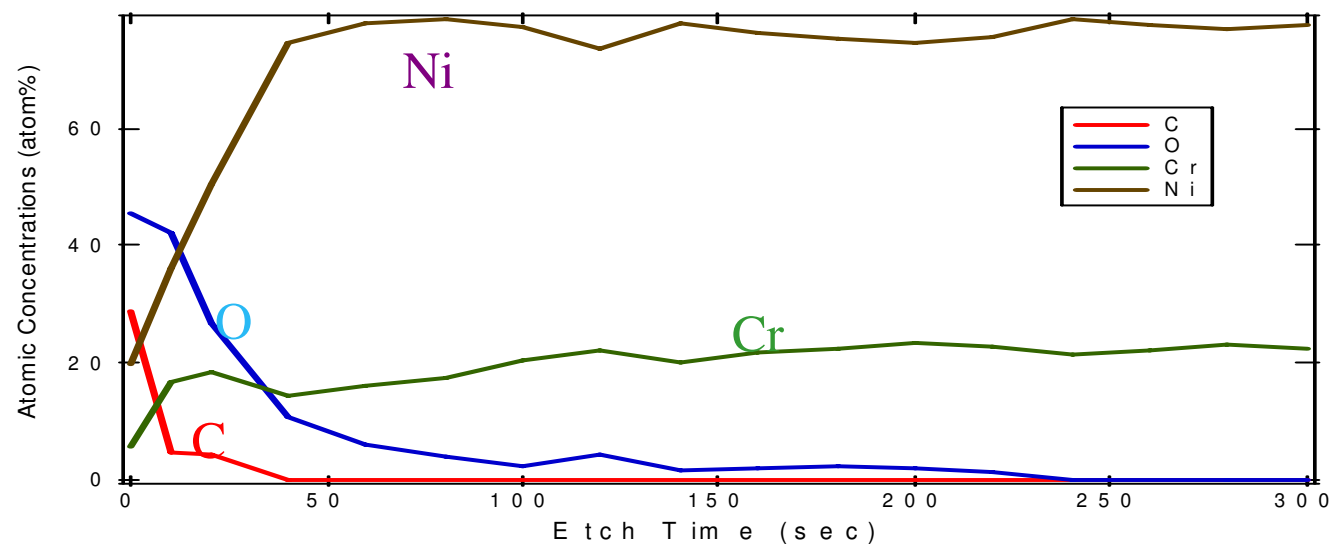

FIG 3. XPS depth profile traces, oxygen is only detected as a surface contaminant.
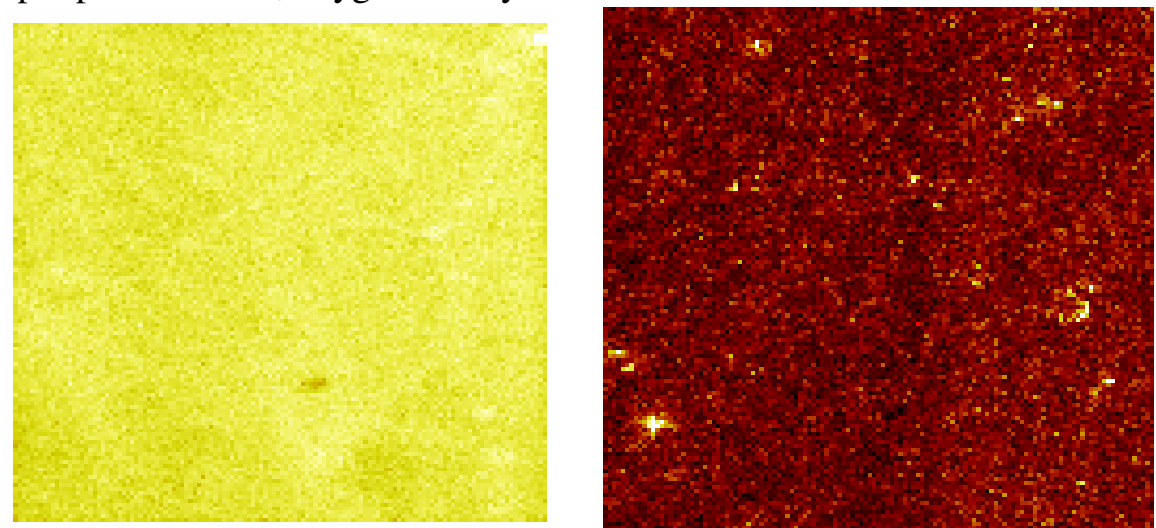

FIG 4. ToF-SIMS images of the sample (left) as well as a control (right). The ToF-SIMS images are displayed using a thermal intensity scale where pixels appearing bright have high intensity. 